Research Paper

\title{
Lycium Barbarum Polysaccharides Decrease Hyperglycemia-Aggravated Ischemic Brain Injury through Maintaining Mitochondrial Fission and Fusion Balance
}

\author{
Wen-Jing Liu*, Hai-Feng Jiang*, Faisal UL Rehman*, Jing-Wen Zhang, Yue Chang, Li Jing ${ }^{\bowtie}$, Jian-Zhong \\ Zhang ${ }^{凶}$
}

School of Basic Medical Science, Ningxia Key Laboratory of Cerebrocranial Diseases-Incubation Base of National Key Laboratory, Ningxia Medical University, Yinchuan 750004, China

*Wen-Jing Liu, Hai-Feng Jiang and Faisal UL Rehman have contributed equally to this study and share first authorship.

$\square$ Corresponding authors: Jian-Zhong Zhang, Current Address: Department of Pathology, Ningxia Medical University, Yinchuan 750004, China. zhangjz@nxmu.edu.cn; Li Jing, Current Address: Department of Pathology, Ningxia Medical University, Yinchuan 750004, China. jingli@nxmu.edu.cn

(c) Ivyspring International Publisher. This is an open access article distributed under the terms of the Creative Commons Attribution (CC BY-NC) license (https:// creativecommons.org/licenses/by-nc/4.0/). See http://ivyspring.com/terms for full terms and conditions.

Received: 2016.11.18; Accepted: 2017.04.14; Published: 2017.07.07

\begin{abstract}
Although it has been reported that polysaccharides found in Lycium barbarum possess neuroprotective effects, little is known of their ability to ameliorate hyperglycemia-aggravated ischemia/reperfusion brain injury. In this study, normoglycemic (NG) and hyperglycemic (HG) rats were compared after 30 minutes of middle cerebral artery occlusion (MCAO), followed by 24 or 27 hours of reperfusion, with HG rats pretreated with lyceum barbarum polysaccharides (LBP) or insulin. In each group, the neurological deficit, infarct volume, pathohistology, and expression of proteins, Opal and Drpl, were assessed to determine the efficacy of LBP in alleviating hyperglycemia-aggravated ischemia/reperfusion brain injury. Our results show that, compared to the NG group, the HG group had increases in neurological deficits, infarct volume, and evidence of neuronal pyknosis at 24- and/or 72-h of reperfusion $(P<0.05)$ and that pre-treatment with LBP decreased these effects $(P<0.05)$. In addition, immunohistochemistry revealed an increase of Drpl and a decrease of Opal positive neurons in the HG group after 24 and 72 hours of reperfusion when compared to the NG group. LBP treatment prevented the HG-induced alterations in Drp-1 and Opal expression. Western blots further confirmed these findings showing that HG caused an increase in phospho-Drpl and a decrease in Opal which were subsequently reversed by LBP addition. These results suggest that hyperglycemia-aggravated ischemic brain damage is associated with an alteration of mitochondrial dynamics and that pre-treatment with LBP ameliorates the hyperglycemia-enhanced ischemic brain damage through maintaining mitochondrial dynamic balance.
\end{abstract}

Key words: Lycium barbarum polysaccharides; hyperglycemia; neuroprotection; cerebral ischemia and reperfusion; mitochondrial fission/fusion; dynamin-related protein 1; optic atrophy 1.

\section{Introduction}

Lycium barbarum, commonly known as Chinese wolfberry or Goji Berry, is a plant belonging to the Solaneae Reich, Solanaceae family. It has been used as a traditional Chinese medicine for over two-thousand years in part to increase visual acuity and to prevent liver damage. In recent years, it has also been used to reduce the side effects of chemotherapy and radiotherapy [1]. Recent studies have shown that Lycium barbarum polysaccharides (LBP) are the main active components in Chinese wolfberry fruit and are 
responsible for an antioxidant function observed in Lycium barbarum $[1,2,3]$. LBP has also been shown to protect neurons from ischemic retinal injury, ischemic brain injury, and beta-amyloid peptide-induced neuronal cell death [4, 5, 6, 7]. However, its mechanism of function and the molecular pathways involved remain elusive $[1,2]$.

Cerebrovascular diseases are a common cause of death in humans and the primary reason for disabilities in elderly populations [8]. Contributing to these outcomes, clinical studies have shown that underlying conditions, such as diabetes or high blood glucose levels, could significantly aggravate brain injury after stroke [9]. Several animal models have confirmed that hyperglycemia increases cerebral infarct volume and cerebral hemorrhage, leading to a poorer prognosis [10]. However, the mechanisms of hyperglycemia-exacerbated

cerebral ischemia/reperfusion damage are still not fully understood and there is no appropriate therapeutic approach to prevent or control the hyperglycemia-enhanced ischemic brain damage [11]. It has been reported that hyperglycemia increases neuronal death through activation of mitochondria-initiated apoptotic pathways [12]. Mitochondrial dynamics is closely related to apoptosis [13]. For example, mitochondrial dynamic imbalance towards mitochondrial fission promotes cytochrome c (Cyto-C), apoptosis-inducing factor (AIF) and second mitochondria-derived activator of caspases (SMAC) release from mitochondria into the cytoplasm, which in turn activates the mitochondria-mediated apoptosis pathways [13]. Therefore, mitochondrial dysfunction and membrane stability play an important role in ischemic brain injury [14]. Hyperglycemia-aggravated cerebral ischemic injury may be related to mitochondrial dynamic imbalance. The aim of the present study is to examine the protective effect of the LBP on hyperglycemia-aggravated ischemia/reperfusion brain injury and its relationship to mitochondrial dynamics and mitochondria-initiated cell death pathways. To that end, streptozotocin (STZ)-induced diabetic hyperglycemic rats were subjected to $30 \mathrm{~min}$ of MCAO, followed by 24 and 72 hours of reperfusion. We observed histopathological outcomes and measured the levels of optic atrophy 1 (Opa1) and dynamin-related protein 1 (Drp1) using immunohistochemistry and Western blot. These findings were compared to normoglycemic and hyperglycemic with either LBP or insulin pre-treatment before MCAO and reperfusion.

\section{Materials and Methods}

\section{Animals and reagents}

Sprague-Dawley rats (8 weeks old, 200-220g) were provided by the Experimental Animal Center of Ningxia Medical University (Yinchuan, China). All animal use and procedures were in strict accordance with the Chinese Laboratory Animal Use Regulations. Efforts were made to minimize animal stress and to reduce the number of rats used for this study. The following chemicals and antibodies were used: 2,3,5-triphenyltetrazolium chloride (TTC, Sigma, USA), monoclonal Opa1 antibody (Abcam, Hong Kong), monoclonal Drp1 antibody (Abcam, Hong Kong), phospho-Drp1 antibody (Cell Signaling Technology, MA, USA), horseradish peroxidase-conjugated secondary antibody (Sigma, USA), streptozotocin (STZ, Sigma, USA), Lycium Barbarum polysaccharides (LBP, Ningxia Agricultural College, Yinchuan, China). Opa1 has one or two cleavage sites (S1 and S2) on the N-terminus and the average value of 3 bands were analyzed using Western blot.

\section{Animal groups}

The rats were randomly assigned to four groups (Table 1), (1) MCAO in normoglycemic rats (NG); (2) $\mathrm{MCAO}$ in hyperglycemic rats (HG); (3) MCAO in hyperglycemic rats pretreated with LBP (LBP); (4) MCAO in hyperglycemic rats pretreated with insulin (Insulin). Each group was further divided into 3 subgroups according to reperfusion time-points (sham, 24- and 72-h).

Table 1. Summary of groups and sub-groups

\begin{tabular}{lllll}
\hline Groups & Processing & TTC & Histo/IHC & WB \\
\hline NG & & 7 & 12 & 8 \\
Sham & Saline+ sham & 2 & 2 & 2 \\
R24-h & Saline+ MCAO & 5 & 5 & 3 \\
R72-h & Saline+ MCAO & 0 & 5 & 3 \\
HG & & 7 & 13 & 9 \\
Sham & STZ + saline+sham & 2 & 3 & 3 \\
R24-h & STZ + saline+MCAO & 5 & 5 & 3 \\
R72-h & STZ + saline+MCAO & 0 & 5 & 3 \\
LBP & & 7 & 13 & 9 \\
sham & STZ +LBP+sham & 2 & 3 & 3 \\
R24-h & STZ +LBP+MCAO & 5 & 5 & 3 \\
R72-h & STZ +LBP+MCAO & 0 & 5 & 3 \\
Insulin & & 7 & 13 & 9 \\
sham & STZ +insulin+sham & 2 & 3 & 3 \\
R24-h & STZ +insulin+MCAO & 5 & 5 & 3 \\
R72-h & STZ +insulin+MCAO & 0 & 5 & 3 \\
\hline
\end{tabular}

R24-h and R72-h: reperfusion at 24-h and 72-h; MCAO: middle cerebral artery occlusion; Histol: histology; IHC: immunohistochemistry; WB: Western blot. 


\section{Induction of diabetes}

Diabetes was induced by i.p. injection of streptozotocin (STZ, $60 \mathrm{mg} / \mathrm{kg}$ ) freshly dissolved in citrate buffer ( $\mathrm{pH}$ 4.5). The blood glucose was measured with a Blood Glucose Meter (Boshilong, Taiwan) after 72-h of STZ injection. The blood glucose levels $>16.8 \mathrm{mmol} / \mathrm{L}$ was assigned to diabetic group [15].

\section{Drug treatments}

Animals in LBP-treatment group were injected daily i.p. with LBP dissolved in saline (25 $\mathrm{mg} / \mathrm{kg} /$ day). LBP treatments were initiated 3 weeks after the induction of diabetes and continued for 4 weeks (w) prior to the induction of MCAO. Animals in the Insulin group were treated with insulin (2 $\mathrm{U} /$ day) by subcutaneous injections following the same protocol as in the LBP group. The Insulin treatment serves as a standard control. The rats in the NG and the sham subgroups of HG groups received saline (i.p.) for 4 weeks.

\section{MCAO and sample preparations}

The animals were anesthetized with 3\% isoflurane for induction and maintained at $1.0-1.5 \%$ during the surgical procedures [16]. The anesthesia was delivered in a $70 \% \mathrm{~N}_{2} \mathrm{O} / 30 \% \mathrm{O}_{2}$ mixture using a facemask (MATRX VIP 3000). The rats underwent right MCAO to induce focal cerebral ischemia. Briefly, the internal carotid artery (ICA), external carotid artery (ECA), and the common carotid artery (CCA) were isolated through a midline incision. The right ECA and CCA were ligated at the proximal end away from the bifurcation. A small incision was made on the ECA and a nylon filament (Sunbio Biotech CO., Beijing) was inserted from the ECA to ICA until a faint resistance was felt. After $30 \mathrm{~min}$ occlusion, the filament was withdrawn to achieve recirculation. After surgery, the rats were maintained in an air-conditioned room at $20^{\circ} \mathrm{C}$ within an animal intensive care unit (Lyon). In the sham group, the blood vessels were separated but without occlusion of the MCA. Neurobehavioral performances were evaluated after the animals woke up and rats with a Zea-Longa scores [16] of 2-3 were regarded as a successful occlusion model.

At 24- and 72-h of reperfusion after $30 \mathrm{~min}$ ischemia, the rats were re-anaesthetized. Animals used for histology were perfusion fixed with $4 \%$ paraformaldehyde and the brains post-fixed in $4 \%$ paraformaldehyde for $24 \mathrm{~h}$ at room temperature. The brains were cut into $2 \mathrm{~mm}$ thick coronal blocks and embedded in paraffin. The blocks were sectioned into $4 \mu \mathrm{m}$ slices and used for Hematoxylin \& Eosin (HE) staining and immunohistochemistry. Animals used for protein assays were euthanized and the brains from the ischemic caudoputamen and the overlaying cortex were stored at $-80^{\circ} \mathrm{C}$ for Western blotting.

\section{Infarct volume assessment}

The brains were removed and frozen at $-20^{\circ} \mathrm{C}$ and were subsequently cut into $2 \mathrm{~mm}$ thick coronal sections. These sections were immersed in a $1.5 \%$ solution of TTC at $37^{\circ} \mathrm{C}$ in a thermostatic incubation box for 30 min, turning back and forth once every 15 min with ophthalmic forceps and fixed in $4 \%$ formaldehyde overnight. Image-J image analysis software 1.48 was used to measure infarct volume (pale region). The infarct volume was calculated according to the following equation: (contralateral area - ipsilateral non-infarct area) / contralateral area x $100 \%$ [16].

\section{Neurological deficits score}

Neurological deficits were scored using a modified scoring system developed by Zea-Longa [16]: 0 , no neurological deficits; 1 , failure to fully extend right paw; 2 , circling to the right; 3 , falling to the right; 4, unable to walk spontaneously and exhibiting depressed levels of consciousness. The rats with scores of 2 - 3 were selected as the MCAO models. The T-maze was used to track animal behavior, which recorded movement from the approach alley to the goal arms, latency, number of crossings, and residence time in the right arm. At the beginning of the test, the rats were placed in the initial arm and were allowed to freely choose the target arm after opening the small gate. No matter which side the animal chose, it received a food reward. Then, in a subsequent test, if the rat entered a goal arm that it had not previously entered, it received a reward; the times were recorded during all tests. Each rat was tested five individual times.

\section{Immunohistochemistry}

For immunohistochemistry, the 4 - $\mu$ m-thick sections were submerged in citrate buffer $(\mathrm{pH}=6.0)$ and heated at boiling temperature under a high-pressure situation for $5 \mathrm{~min}$ for antigen retrieval. To block endogenous peroxidase activity, the sections were allowed to cool to room temperature and were treated with $3 \% \mathrm{H}_{2} \mathrm{O}_{2}$ for $30 \mathrm{~min}$ at room temperature and blocked by $5 \%$ bovine serum albumin (BSA, in PBS). The sections were incubated overnight $\left(4^{\circ} \mathrm{C}\right)$ with primary antibodies specific to Drp1 (1:200) and Opa1 (1:200). The sections were washed in phosphate-buffered saline (PBS) and then incubated in horseradish-peroxidase-conjugated anti-rabbit secondary antibody for $1 \mathrm{~h}\left(37^{\circ} \mathrm{C}\right)$. The reaction was visualized with diaminobenzidine (DAB). Images from five microscopic fields $(400 \times, \mathrm{HPF})$ were 
captured with a microscope and analyzed with Image-J analysis software 1.48. Number of positively stained cells was counted with the average number of 5 different microscopic fields.

\section{Western blot}

The frozen brain tissues from the ischemic caudoputamen and the corresponding cortex were homogenized on ice with lysis buffer. All protein samples from the different groups $(50 \mu \mathrm{g} / \mu \mathrm{l})$ were electrophoresed on an $8 \%$ sodium dodecyl sulfate polyacrylamide electrophoresis gel and were then electro-transferred to polyvinylidene fluoride membranes $(0.45 \mu \mathrm{m}$, Millipore, USA). The membranes were incubated in 5\% BSA for $3 \mathrm{~h}$ at room temperature and were then immersed in primary antibody overnight at $4^{\circ} \mathrm{C}$. The primary antibodies were as follows: Rabbit anti-Opa1 antibody (1:2000), Rabbit anti-Drp1 antibody (1:2000), Rabbit anti-phospho-Drp1 antibody (1:1000), and anti- $\beta$-tubulin (1:2000). On the following day, the membranes were washed with tris buffered saline and Tween-20 ( TBST, pH 8.0) and incubated in secondary antibody (peroxidase-conjugated AffiniPure goat anti-mouse IgG or goat anti-rabbit IgG, 1:5000) for $1 \mathrm{~h}$ at room temperature. The signal protein bands were detected by chemical luminescence (Thermo Fisher, USA) and the images were captured using the Gel Imaging Analysis System (Amersham Imager 600, GE Healthcare). Semi-quantitative results were obtained by measuring the optical density of the target bands and were expressed as the ratio of each targeted protein to $\beta$-tubulin.
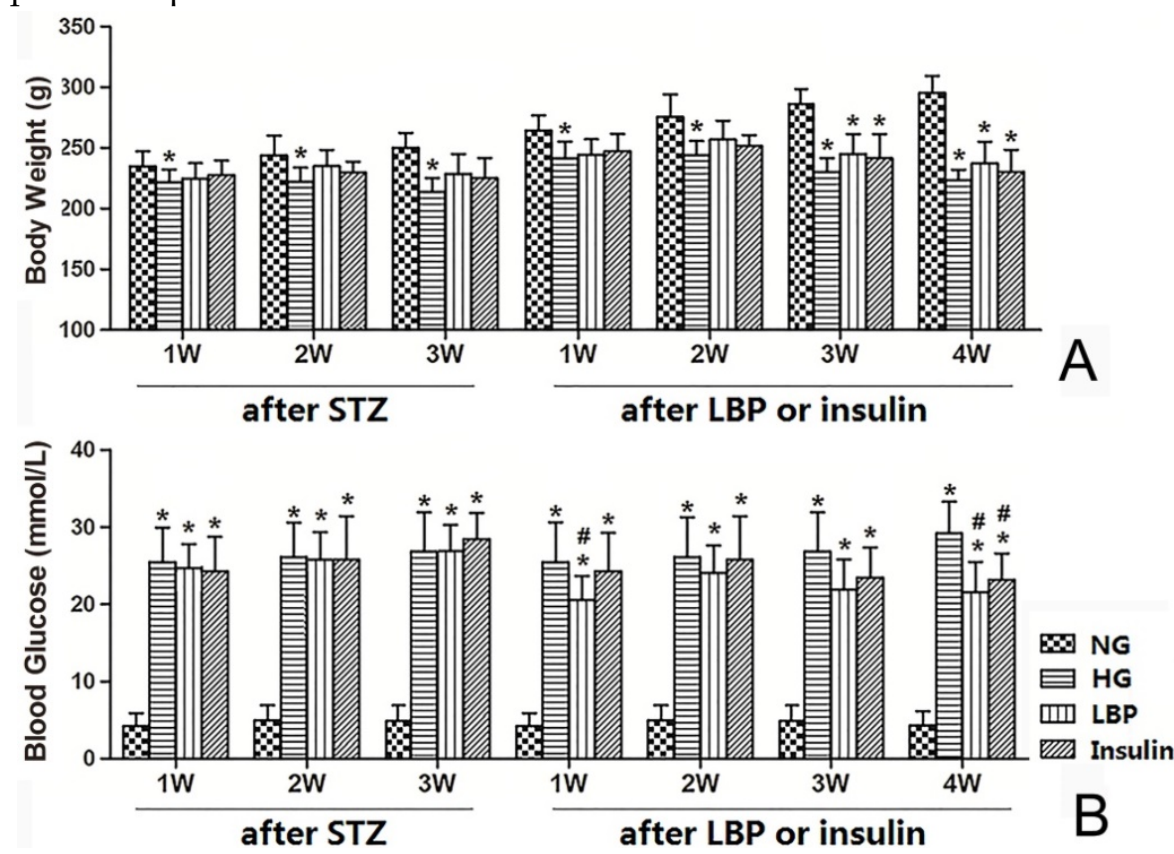

Figure 1. Body weight and blood glucose changes in NG, HG, LBP, and Insulin groups after STZ administration and after LBP or insulin therapy. A, Summary of body weights; B, Summary of blood glucose levels. $* P<0.05$, vs. NG group; $\# P<0.05$, vs. HG group, respectively.

\section{Statistical analysis}

All data were expressed as mean \pm standard deviation (SD). Statistical analysis was performed using one-way ANOVA with SPSS 19.00. Tukey's post hoc test was used for multiple comparisons. The $t$-test was used for testing between two groups. Statistical significance was determined as $P<0.05$.

\section{Results}

\section{Body Weight and blood glucose}

The HG groups had lower body weights than the NG group after 1 week of diabetes (Fig. $1 \mathrm{~A}, P<0.05$ ). The body weight of rats in LBP and insulin treated groups were also lower than the NG group at $3-$ and 4 - weeks of LBP or insulin-injections $(P<0.05)$. However, there were no significant differences in body weight among LBP, Insulin and HG groups (Fig. 1A). As expected, the blood glucose levels were significantly increased in the HG, LBP, and Insulin groups after STZ-injection (Fig. 1B). The blood glucose levels at 1- and 4- weeks of LBP injections in LBP group showed obvious decreases compared to the HG group $(P<0.05)$. Additionally, the treatment of insulin also lowered the blood glucose level at 4 weeks (Fig. 1B).

\section{Assessments of neurological deficits and T-maze}

Neurological deficits were not found in sham-operated animals. Neurological deficits were observed at 24- and 72-h of reperfusion in the NG, HG, LBP, and Insulin groups as expected (Fig. 2A). Compared with the NG group, the HG group had more severe neurological deficit scores after ischemia/reperfusion $(P<0.05)$. Pre-treatment with LBP or insulin significantly decreased neurological deficit scores at 24and $72-\mathrm{h}$ of reperfusion (Fig. 2A, $P<0.05)$. The T-maze test (Fig. 2B), which measures learning functional deficits and spatial working memory, showed that rats in HG group spent less time than the NG group at the target arm after ischemia/reperfusion $(P<0.05)$, and the LBP and Insulin groups spent longer times there than the HG group at 24- and 72-h of reperfusion $(P<0.05)$. 


\section{Infarct volume}

As expected, no infarct was observed in the sham rats (Fig. 3A). Compared with the NG group, the HG group had larger cerebral infarct volumes (Fig. 3B, P<0.05). LBP and insulin treatment prior to $\mathrm{MCAO}$ resulted in significantly reduced infarct volumes 24 hours after reperfusion $(P<0.05)$.
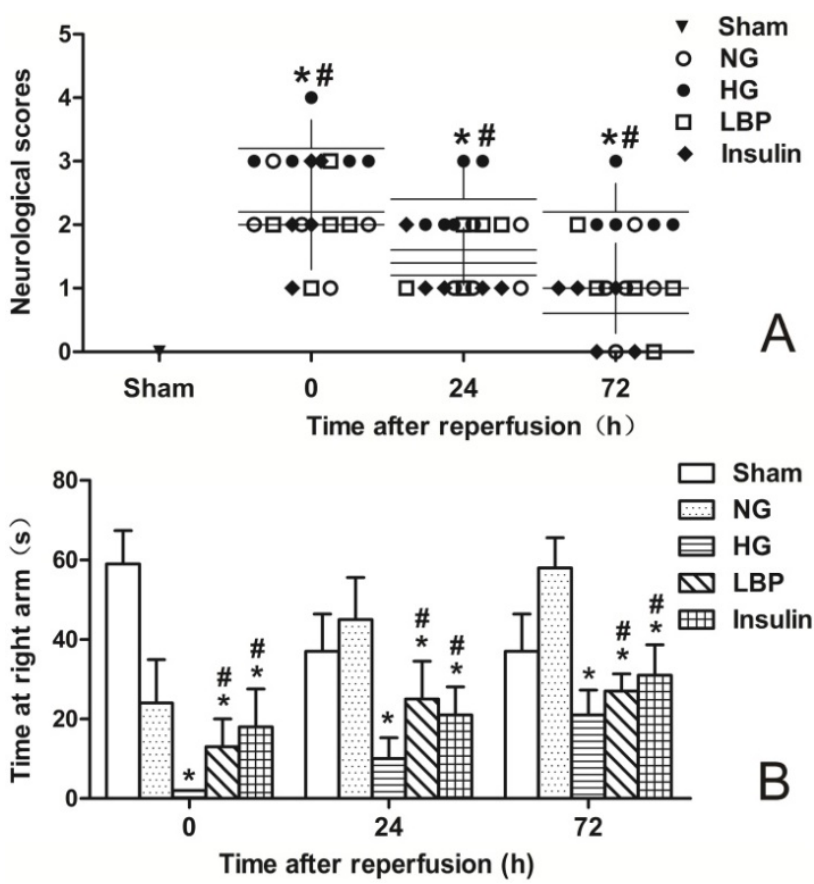

Figure 2. Neurological deficits score and T-maze test in sham, NG, HG, LBP and Insulin groups after MCAO and 24- or 72-h reperfusion. A, Summary of the neurological deficits score; $B$, Summary of the time in T-maze test. $* P<0.05$,vs. NG group; $\# P<0.05$, vs. HG group, respectively.
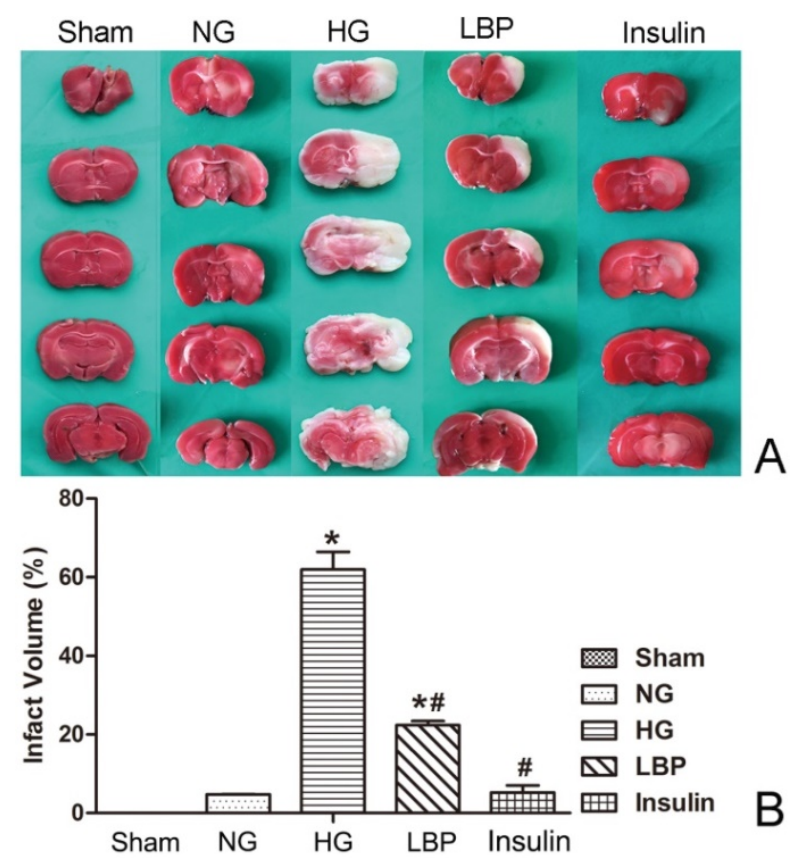

Figure 3. Comparison of infarct volumes after 24 hours reperfusion. A, Representative brain sections stained with TTC. Pale areas show infarct volume of the hemisphere in HG and LBP groups; B, Quantitative summary of infarct volumes. $* P<0.05$, vs. sham group; $\# P<0.05$, vs. $H G$ group, respectively.

\section{Histopathology}

Histopathology was observed in brain tissue from the frontal cortex and caudate nucleus using Hemotoxylin and Eosin stained sections (Fig. 4A). In the NG ischemia group, swollen neurons in the infarct region, widened gaps between cells and small blood vessels, and shrunken neurons were observed (Fig. 4A). At 24- and 72-h reperfusion, the numbers of neuronal pyknosis were significantly increased in the HG group compared to NG group (Fig. 4B, $P<0.05$ ). Compared with the HG group, LBP or insulin pre-treatment reduced neuronal pyknosis at 24- and 72-h reperfusion (Fig. 4B, $P<0.05$ ).

\section{Localized expression of Opal and Drpl}

Abundant positive staining of Opa1 was observed in neurons from brains of sham-operated and NG animals (Fig. 5A). Following ischemia and reperfusion, the numbers of Opa1-positive cells were decreased in NG, HG, LBP, and Insulin groups (Fig. 5B). Conversely, the numbers of Opa1-positive cells were significantly decreased in the HG group when compared to the NG group (Fig. $5 B, P<0.05$ ). LBP or insulin pre-treatment up-regulated Opa1 expression in the cells at 24- and 72-h reperfusion (Fig. 5B, $P<0.05)$.

As shown in Figure 6, Drp1 expression was detected in the cortex of HG sham-operated rats (Fig. 6A). The numbers of Drp1-immunostained cells were increased after ischemia/reperfusion (Fig. 6B). Compared with the NG group, the number of Drp1-positive cells in the HG group was significantly increased at 24- and 72-h reperfusion (Fig. 6B, $P<0.05$ ). The LBP or Insulin groups exhibited significantly decreased Drp1-positive cells at 24- and 72-h reperfusion (Fig. $6 \mathrm{~B}, P<0.05$ ).

\section{Western blot of Opal and Drpl}

As shown in Figure 7, increased expression of Drp1 and phospho-Drp1 and decreased expression of Opa1 were demonstrated by Western blotting in HG group at 24- and 72-h reperfusion when compared to NG ischemic rats (Fig. 7A). Pre-treatment with LBP increased the expression of Opa1 at 24-h reperfusion and suppressed the expression of Drp1 and phospho-Drp1 at 24- and 72-h reperfusion (Fig. 7A). Compared with the NG group, the relative expression of Opa1 was significantly decreased (Fig. 7B), and the ratio of phospho-Drp1/Drp1 was increased in HG group at $24-h$ reperfusion $(P<0.05)$. Meanwhile, compared to HG group, the relative value of Opa1 was significantly increased in LBP group at 24-h reperfusion, and the ratio of phospho-Drp1/Drp1 was decreased in LBP group at 24- and 72-h reperfusion (Fig. 7C, $P<0.05$ ). 


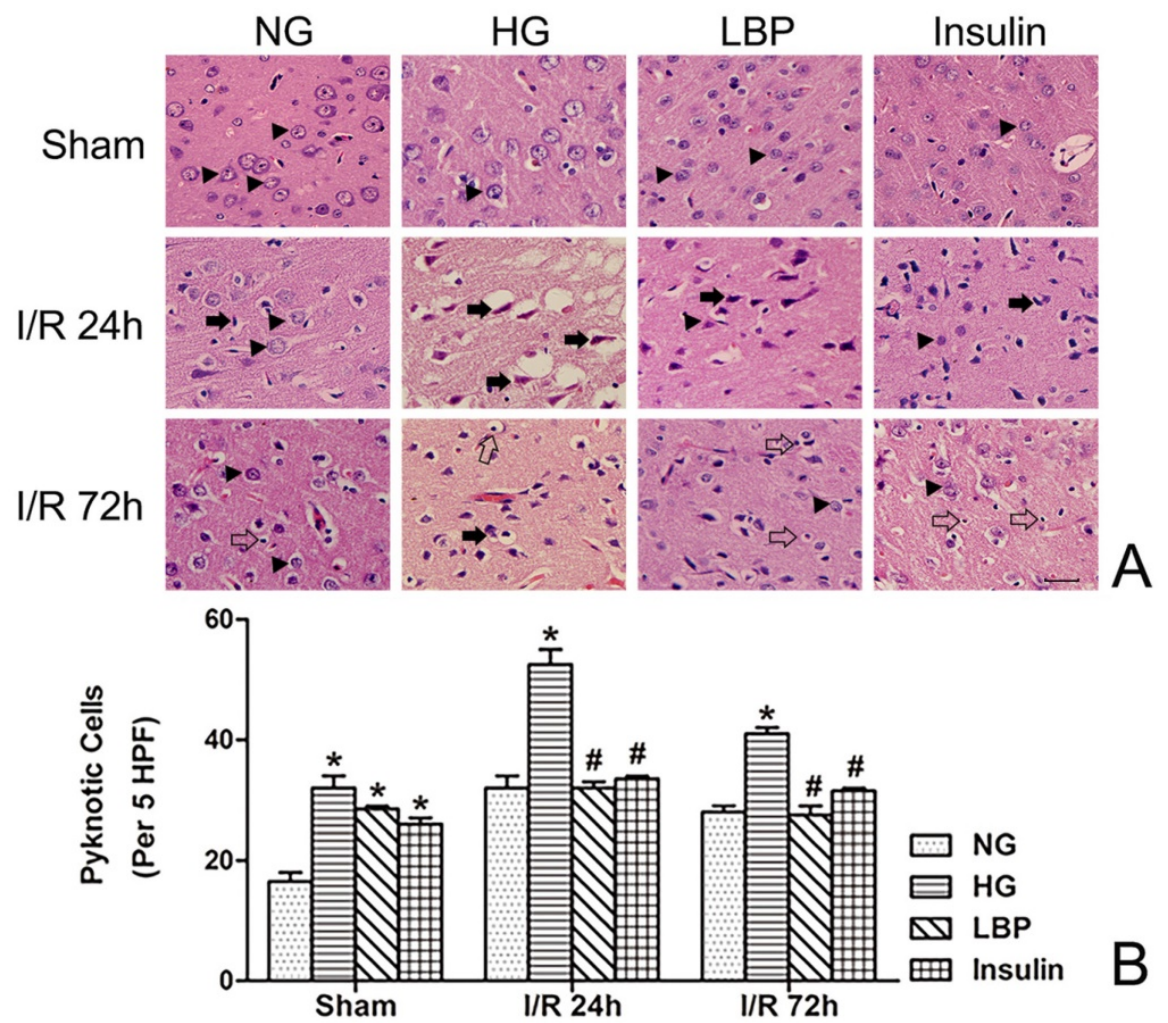

Figure 4. Observed histopathology and changes in number of pyknotic cell in NG, HG, LBP and Insulin groups. A, Representative pictograghs of the cortex after 24 and 72 hours reperfusion. Normal histology and neurons are indicated by arrow heads in the sham groups; solid arrows point to pyknotic and swollen neurons after 24 hours reperfusion; glial cells are indicated by empty arrows and compared at 72 -h reperfusion. Bar $=100 \mu \mathrm{m}$. B, Quantitative summary of pyknotic cells; $* P<0.05$, vs. NG group; $\# P<0.05$, vs. HG group, respectively.

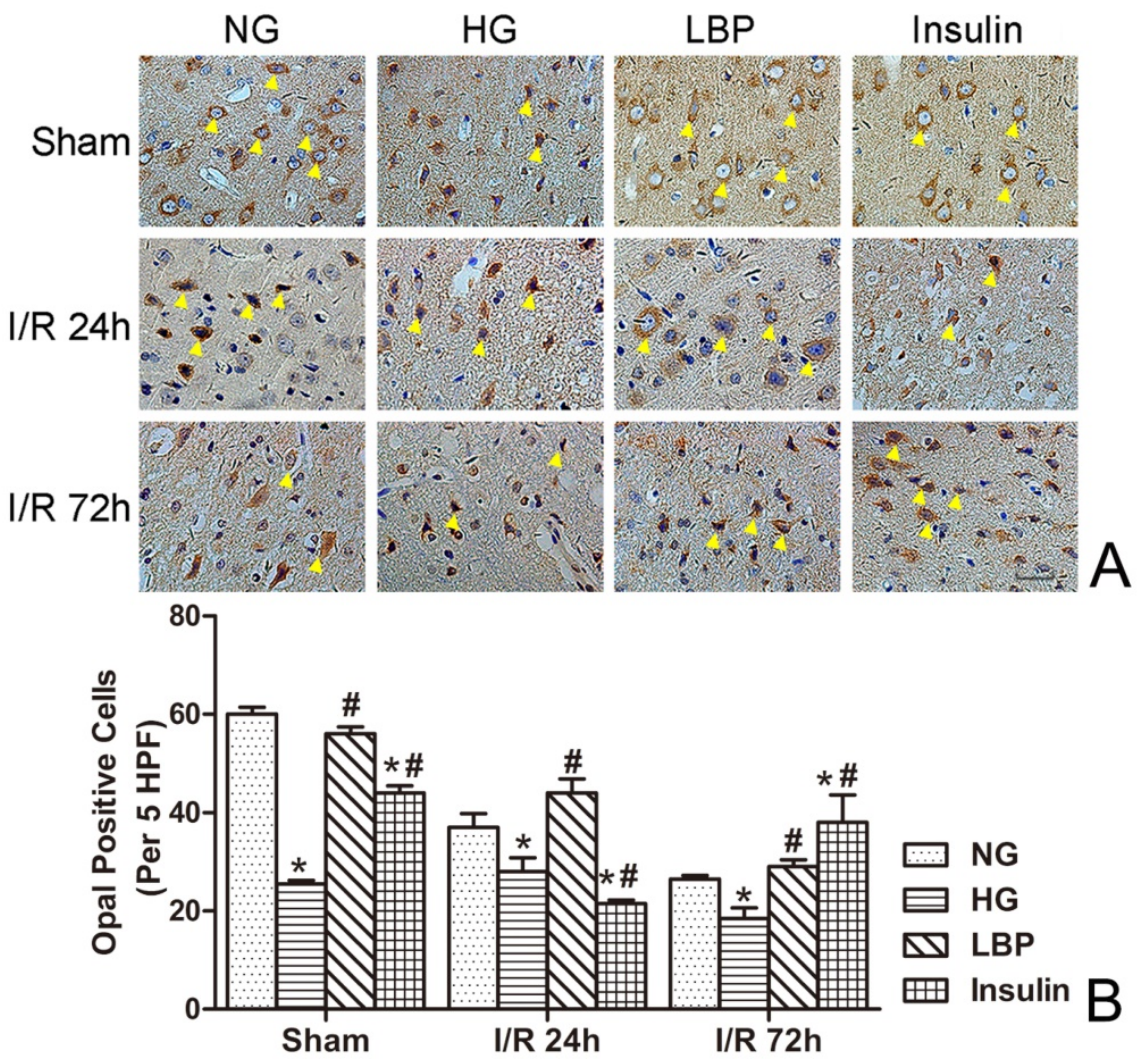

Figure 5. Expression of Opal in NG, HG, LBP, and Insulin groups after 24 and 72 hours of reperfusion. A, Representative pictograghs showing immunohistochemistry staining of Opal. Opal positively stained cells appear brown against a blue counterstain (hematoxylin). Yellow arrow heads indicate positively stained cells. Bar $=50 \mu \mathrm{m}$. B, Quantitative summary showing the number of Opal-positive cells; $* P<0.05$, vs. NG group; \#P<0.05 vs. HG group, respectively. 

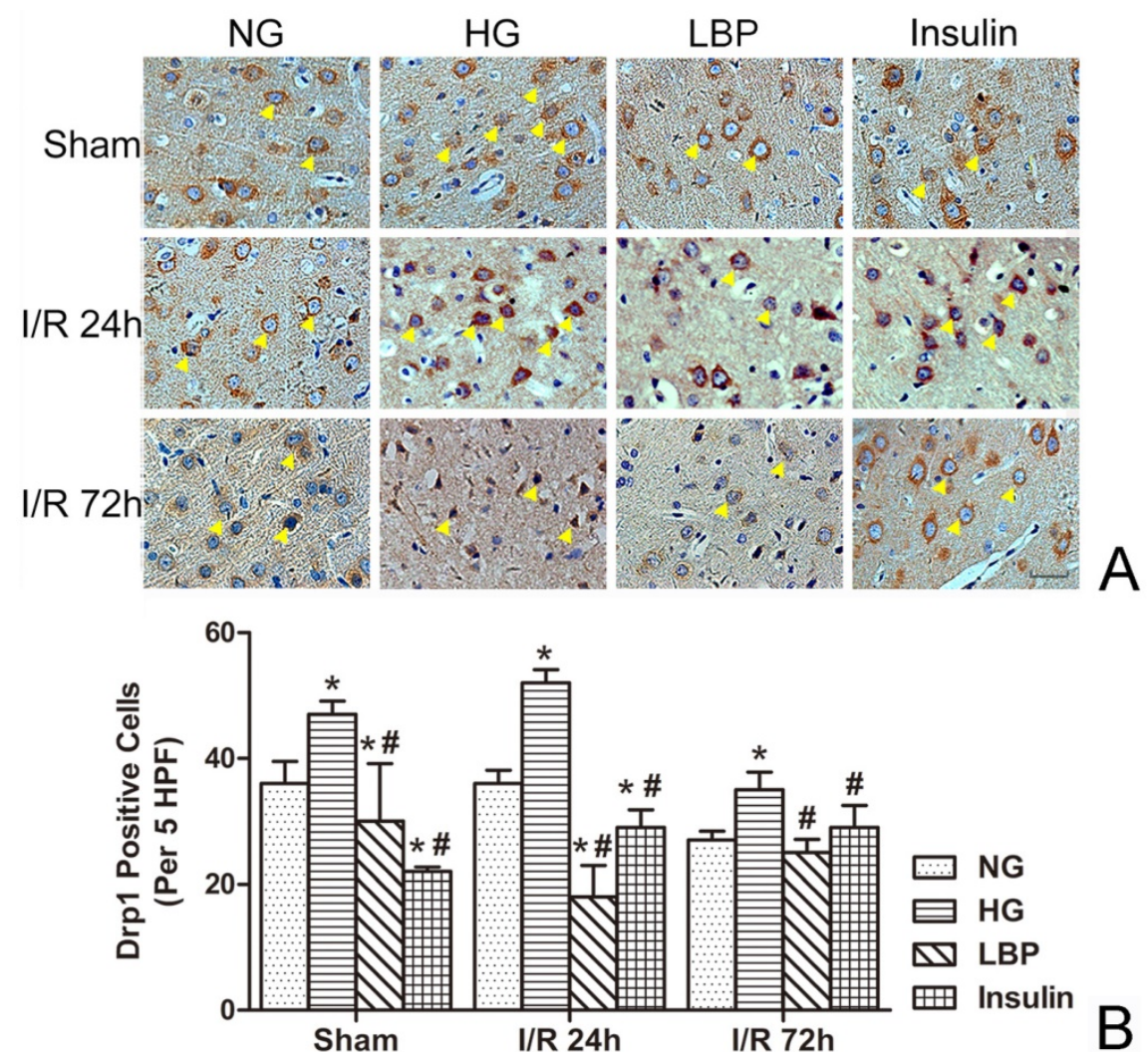

Figure 6. Expression of Drpl in NG, HG, LBP and Insulin groups after 24 and 72 hours of reperfusion. A, Representative pictograghs showing immunohistochemistry staining of Opal. Opal positively stained cells appear brown against a blue counterstain (hematoxylin). Yellow arrow heads indicate positively stained cells. Bar $=50$ $\mu \mathrm{m}$. B, Quantitative summary showing the number of Drpl-positive cells; $* P<0.05$, vs. NG group; $\# P<0.05$, vs. $\mathrm{HG}$ group, respectively.
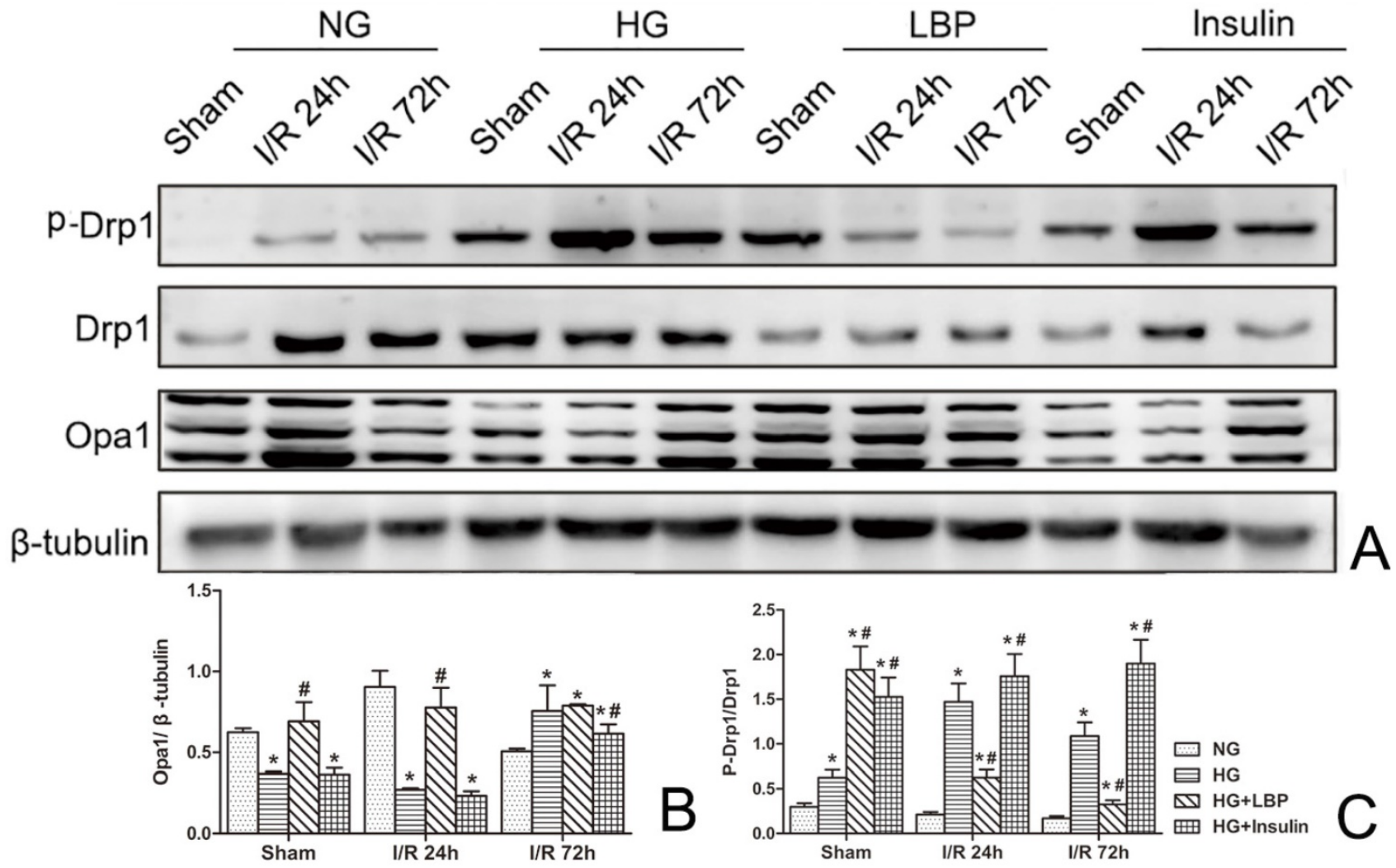

Figure 7. Western blotting analysis of Drpl and Opal after 24 and 72 hours of reperfusion. A, Representative Western blot of Opa 1, Drpl, and phospho-Drp 1; B, Summary of relative values of Opal; C, Summary of the ratio of phospho-Drpl/Drpl; $* P<0.05$, vs. NG group; $\# P<0.05$, vs. HG group, respectively. 


\section{Discussion}

Diabetes is one of the most common diseases in humans, and diabetic hyperglycemia is a major leading factor in tissue damage [8]. Diabetic patients with high blood glucose levels have a greater chance of suffering from stroke, and diabetic patients after stroke have significantly worse neurological outcomes than non-diabetic patients $[8,9]$. In the present study, the diabetic hyperglycemia rat model was successfully established, following $30 \mathrm{~min}$ ischemia and 24- and/or 72-h reperfusion. Rats with high blood glucose concentrations exhibited larger infarction volumes, more neurological deficit scores, reduced T-maze test times, and more pyknotic neurons than normoglycemic rats. These results suggest that diabetic hyperglycemia aggravates brain injury induced by ischemia/reperfusion. The mechanisms of hyperglycemia-aggravated cerebral ischemia/ reperfusion injury are poorly understood, and interventions for hyperglycemia-aggravated ischemic brain injury have made little progress [11,13]. Therefore, future studies are needed to explore the molecular mechanisms of hyperglycemia-aggravated cerebral ischemia/reperfusion injury and potential effective drugs for treating this disease [11].

LBP is the main polysaccharide isolated from the Chinese wolfberry, a plant dominantly grown in the Ningxia district of People's Republic of China, and is probably the active component of the plant $[1,2,17$, 18]. Pharmacological studies have reported that LBP possess anti-oxidant, anti-aging, anti-tumor, anti-hypertension, lipid-lowering, and immuneregulating effects $[1,17]$. In the present study, we demonstrated that pre-treatment with LBP in diabetes-hyperglycemic rats decreased the neurological deficit scores, infarct volumes, and pyknotic neurons compared to the non-treated hyperglycemic ischemic rats at 24- and/or 72-h reperfusion. The results suggest that the pre-treatment with LBP in rats reduces the neurological deficits and neuronal injury induced by diabetic hyperglycemia. Our study has shown that LBP prevents damage to neurons to effectively limit brain damage caused during ischemia/reperfusion with underlying diabetic hyperglycemia. LBP have been shown to increase survival of cultured retinal ganglion cells, as well as to decrease neuronal cell death, retinal swelling, and oxidative injury following retinal ischemia/reperfusion damage $[18,19,20]$. LBP can also increase cell-surface levels of glucose transporter type 4 (GLUT4) in vivo and improve GLUT4 trafficking and intracellular insulin signaling [21]. Furthermore, LBP enhances the survival of damaged pancreatic islets cells, protects pancreatic cells against disturbances, and increases the number of $\beta$-cells to raise insulin secretion [22, 23].

Mitochondria are highly dynamic organelles that experience continuous fission, fusion, trafficking, and turnover, all of which contribute to the maintenance of mitochondrial function [12, 13]. Mitochondrial dynamics contributes to the stability of the mitochondrial membrane, which constitutes a dynamic interconnecting network [13, 14]. The changes in mitochondrial dynamics are regulated by GTP-related proteins, which include mitochondrial fusion protein 1 and 2 (Mfn1/2), Opa1, and Drp1 [13, 14]. Mfn $1 / 2$ is located in the outer membrane and promotes fusion of two adjacent mitochondria. Opa1 is located in the inner mitochondrial membrane, which plays a crucial role in mediating mitochondrial fusion within the inner membrane [14]. Using siRNA to block Opa1 expression results in morphological changes in the mitochondrial cristae, as well as vesicle structure production [24]. Opa1 is also essential for the assembly and stability of the mitochondrial respiratory chain and the sequestration of pro-apoptotic cytochrome molecules within these tight junctions [25]. Conversely, Drp1 is mainly found in the cytoplasm and can be transferred from the cytoplasm to the outer mitochondrial membrane, where Drp1 combines with Fis1 in the outer membrane of the mitochondria for mitochondrial fission [26]. These two factors work together to form a loop in the middle of mitochondria and divides them into two parts [26]. Mitochondrial fission is mainly controlled through Drp1 activity, and the frequency of the split determines mitochondrial morphology and function [26, 27]. Therefore, Drp1 is essential for the stabilization of mitochondrial morphology within the cells $[27,28]$. In the present study, we have shown that transient focal cerebral ischemia/reperfusion down-regulated Opa1 and up-regulated Drp1 in the normoglycemic rats, suggesting mitochondrial dynamic imbalance towards fission is involved in mediating ischemic neuronal damage. Diabetic hyperglycemia further down-regulated Opa1 and up-regulated Drp1 after MCAO, suggesting that diabetes further tilts the mitochondrial dynamic towards fission.

Numerous studies have focused on mitochondrial fission/fusion in ischemia/reperfusion $[29,30]$. The loss in a balance of fusion and fission can lead to other acute cerebral injuries, especially ischemic stroke [20,29]. In the present study, diabetic hyperglycemia down-regulated Opa1 and up-regulated Drp1 in the temporarily focal cerebral ischemia/reperfusion rat model. LBP intervention can increase the expression of the mitochondrial fusion factor, Opa1, and decrease the expression of the 
mitochondrial cleavage factor, Drp1. These results suggest that LBP may be a mitochondrial membrane stabilizer, as well as play a role in the maintenance of mitochondrial structure in the diabetic hyperglycemic rat models. Previous studies have shown that hyperglycemia aggravates ischemia/reperfusion injury through many signal transduction pathways $[20,31]$. Hyperglycemia increases the accumulation of lactic acid in HT22 cells, which results in the production of alcoholic lactic acidosis, free radicals, and oxidative stress, and also induces brain injury by increasing oxidative damage [20,32]. Hyperglycemia also increases brain damage by activating the mitochondrial apoptotic pathway [13]. Our present research showed that LBP can be a mitochondrial membrane stabilizer through up-regulating Opa1 and down-regulating Drp1. This may suggest a self-protection mechanism of LBP in the mitochondria [33]. This may also be one of the manifestations of LBP many functions, such as its protective role in neurons [4], and anti-oxidant [3, 4] and immune-regulating effects [7].

The limitation of this study is that it provided an association relationship among ischemic brain damage, mitochondrial fission markers and LBP treatment. In order to further clarify the role of mitochondrial dynamic balance in diabetic ischemia, future study could use therapeutic compounds to inhibit mitochondrial fission and to promote mitochondrial fusion.

In summary, in the present study, a diabetic hyperglycemia and temporary focal cerebral ischemia/reperfusion rat model was successfully established. As expected, the blood glucose levels were significantly increased in the HG, LBP, and Insulin groups after STZ-injection $(P<0.05)$. Compared with the NG group, the HG group had increased neurological deficits and neuronal pyknosis at 24- and 72-h $(P<0.05)$. Pre-treatment with LBP and insulin decreased neurological deficits and neuronal pyknosis at 24- and $72-\mathrm{h}$ of reperfusion $(P<0.05)$. Compared with the NG group, the HG groups produced larger cerebral infarct volumes $(P<0.05)$. LBP and insulin treatment prior to MCAO resulted in reduced infarct volumes at 24-h after reperfusion $(P<0.05)$. In immunohistochemistry, Drp1 positive cells were increased and Opa1 positive cells decreased in the HG group at 24- and 72-h reperfusion compared to NG group; Drp1 and Opa1 positive cells in LBP group were decreased and increased, respectively. In Western blotting, a decreased relative value of Opa1 at 24-h reperfusion and an increased ratio of phospho-Drp1/Drp1 at 24- and 72-h reperfusion were demonstrated in HG group compared to NG group; an increased Opa1 and a decreased ratio of phospho-Drp1/Drp1 were tested in LBP group compared to HG group $(P<0.05)$. Overall, these results suggest that hyperglycemia aggravates brain injury induced by ischemia/reperfusion, and pre-treatment with LBP could prevent the enhanced damage through improved mitochondrial dynamics. LBP may be a promising drug for intervention in hyperglycemia-aggravated cerebral ischemic injury.

\section{Acknowledgements}

This work was supported by the National Natural Science Foundation of China (81360184) for Jian-Zhong Zhang, and the National Natural Science Foundation of China (81260184, 81560208) for Li Jing. The authors thank Jian-Da Dong, Yi Ma, Feng-Ying Guo, and Wen Yang for work relating to the setup of the MCAO model and Dr. Mary Zimmerman for editing and proof-reading.

\section{Author Contributions}

Conceived and designed the experiments: JZZ, LJ. Performed the experiments: WJL, HFJ, FULR. Contributed reagents/materials/analysis tools: WJL, JWZ, YC. Wrote the paper: WJL, FULR, LJ, JZZ.

\section{Competing Interests}

The authors have declared that no competing interest exists.

\section{References}

1. Jiao R, Liu Y, Gao H, et al. The anti-oxidant and antitumor properties of plant polysaccharides. Am J Chin Med. 2016; 44(3):463-88.

2. Yang $X$, Bai $H$, Cai $W$,et al. Lycium barbarum polysaccharides reduce intestinal ischemia/reperfusion injuries in rats. Chem Biol Interact. 2013; 204(3):166-72.

3. Zhang R, Kang KA, Piao MJ, et al. Cytoprotective effect of the fruits of Lycium chinense Miller against oxidative stress-induced hepatotoxicity. J Ethnopharmacol. 2010; 130(2):299-306.

4. Li SY, Yang D, Yeung CM, et al. Lycium barbarum polysaccharides reduce neuronal damage, blood-retinal barrier disruption and oxidative stress in retinal ischemia/reperfusion injury. PLoS One. 2011; 6(1):e16380.

5. Yu MS, Lai CS, Ho YS, et al. Characterization of the effects of anti-aging medicine Fructus lycii on beta-amyloid peptide neurotoxicity. Int J Mol Med. 2007; 20(2):261-8.

6. Xing X, Liu F, Xiao J, et al. Neuro-protective mechanisms of Lycium barbarum. Neuromolecular Med. 2016; 18(3):253-63.

7. Wang T, Li Y, Wang Y, et al. Lycium barbarum polysaccharide prevents focal cerebral ischemic injury by inhibiting neuronal apoptosis in mice. PLoS One. 2014; 9(3):e90780.

8. Hafez S, Coucha M, Bruno A, et al. Hyperglycemia, acute ischemic stroke, and thrombolytic therapy. Transl Stroke Res. 2014; 5(4):442-53.

9. Ergul A, Kelly CA, Abdalla M, et al. Cerebrovascular complications of diabetes: focus on stroke. Endocr Metab Immune Disord Drug Targets. 2012; 12(2):148-58.

10. $\mathrm{Li} \mathrm{W}, \mathrm{Qu} \mathrm{Z}$, Prakash R, et al. Comparative analysis of the neurovascular injury and functional outcomes in experimental stroke models in diabetic Goto-Kakizaki rats. Brain Res. 2013; 1541:106-14.

11. Zhang Z, Yan J, Shi H. Hyperglycemia as a risk factor of ischemic stroke. J Drug Metab Toxicol. 2013; 4(4):153.

12. Ten V S, Starkov A. Hypoxic-ischemic injury in the developing brain: the role of reactive oxygen species originating in mitochondria. Neurol Res Int. 2012;2012:542976.

13. Rehni AK, Nautiyal N, Perez-Pinzon MA, et al. Hyperglycemia / hypoglycemia-induced mitochondrial dysfunction and cerebral ischemic damage in diabetics. Metab Brain Dis. 2015; 30(2):437-47.

14. Wu S, Zhou F, Zhang Z, et al. Mitochondrial oxidative stress causes mitochondrial fragmentation via differential modulation of mitochondrial fission-fusion proteins. FEBS J. 2011; 278(6):941-54. 
15. Szkudelski T. Streptozotocin-nicotinamide-induced diabetes in the rat. Characteristics of the experimental model. Exp Biol Med (Maywood). 2012; 237(5):481-90.

16. Longa EZ, Weinstein PR, Carlson $\mathrm{S}$, et al. Reversible middle cerebral artery occlusion without craniectomy in rats. Stroke. 1989; 20(1):84-91.

17. Ho YS, Yu MS, Yang XF, et al. Neuroprotective effects of polysaccharides from wolfberry, the fruits of Lycium barbarum, against homocysteine-induced toxicity in rat cortical neurons. J Alzheimers Dis. 2010; 19(3):813-27.

18. Yang M, Gao N, Zhao Y, et al. Protective effect of Lycium barbarum polysaccharide on retinal ganglion cells in vitro. Int J Ophthalmol. 2011; $4(4): 377-9$.

19. Zhu J, Liu W, Yu J, et al. Characterization and hypoglycemic effect of a polysaccharide extracted from the fruit of Lycium barbarum L. Carbohydr Polym. 2013; 98(1):8-16.

20. Chen $\mathrm{R}, \mathrm{Li} \mathrm{YX}, \mathrm{Hao} \mathrm{YJ}$, et al. Protective effects of Lycium barbarum polysaccharide on neonatal rat primary cultured hippocampal neurons injured by oxygen-glucose deprivation and reperfusion. J Mol Histol. 2012; 43(5):535-42.

21. Zhao R, Qiu B, Li Q, et al. LBP-4a improves insulin resistance via translocation and activation of GLUT4 in OLETF rats. Food Funct. 2014; 5(4):811-20.

22. Tang $\mathrm{L}$, Zhang $\mathrm{Y}$, Jiang $\mathrm{Y}$, et al. Dietary Wolfberry ameliorates retinal structure abnormalities in $\mathrm{db} / \mathrm{db}$ mice at the early stage of diabetes. Exp Biol Med. 2011; 236(6):1051-63.

23. Cai H, Liu F, Zuo P, et al. Practical application of antidiabetic efficacy of Lycium barbarum polysaccharide in patients with type 2 diabetes. Med Chem. 2015; 11(4):383-90.

24. Sanderson TH, Raghunayakula S, Kumar R. Neuronal hypoxia disrupts mitochondrial fusion. Neuroscience. 2015; 301:71-8.

25. Varanita T, Soriano ME, Romanello V, et al. The OPA1-dependent mitochondrial cristae remodeling pathway controls atrophic, apoptotic, and ischemic tissue damage. Cell Metab. 2015; 21(6):834-44.

26. Sharp WW, Fang $\mathrm{YH}, \mathrm{Han} \mathrm{M}$, et al. Dynamin-related protein 1 (Drp1)-mediated diastolic dysfunction in myocardial ischemia-reperfusion injury: therapeutic benefits of Drp1 inhibition to reduce mitochondrial fission. FASEB J. 2014; 28(1):316-26.

27. Huang S, Wang Y, Gan X, et al. Drp1-mediated mitochondrial abnormalities link to synaptic injury in diabetes model. Diabetes. 2015; 64(5):1728-42.

28. Baek SH, Noh AR, Kim KA, et al. Modulation of mitochondrial function and autophagy mediates carnosine neuroprotection against ischemic brain damage. Stroke. 2014; 45(8):2438-43.

29. Kumar R, Bukowski MJ, Wider JM, et al. Mitochondrial dynamics following global cerebral ischemia. Mol Cell Neurosci. 2016; 76:68-75.

30. Bhatti JS, Bhatti GK, Reddy PH. Mitochondrial dysfunction and oxidative stress in metabolic disorders - A step towards mitochondria based therapeutic strategies. Biochim Biophys Acta. 2016; pii: S0925-4439(16)30292-7.

31. Luitse MJ, Biessels GJ, Rutten GE, et al. Diabetes, hyperglycaemia, and acute ischemic stroke. Lancet Neurol. 2012; 11(3):261-71.

32. Kim H, Choi J, Ryu J, et al. Activation of autophagy during glutamate-induced HT22 cell death. Biochem Biophys Res Commun. 2009; 388(2):339-44.

33. Tang Y, Liu X, Zhao J, et al. Hypothermia-induced ischemic tolerance is associated with Drp1 inhibition in cerebral ischemia-reperfusion injury of mice. Brain Res. 2016; 1646:73-83. 